\title{
DWI in transient global amnesia and TIA: proposal for an ischaemic origin of TGA
}

\author{
K Winbeck, T Etgen, H G von Einsiedel, M Röttinger, D Sander
}

J Neurol Neurosurg Psychiatry 2005;76:438-441. doi: 10.1136/jnnp.2004.042432

\begin{abstract}
There are conflicting reports concerning signal intensity changes in transient global amnesia (TGA) using diffusion weighted imaging (DWI). We prospectively analysed DWI signal intensity changes in TIA and TGA patients, and compared the clinical characteristics and risk factors of both groups. Using DWI and conventional T1 and T2 weighted turbo spin echo sequences, 28 patients with acute TGA (13 men, mean age 61.5 years) and 74 TIA patients ( 47 men, mean age 62.4 years) were studied within 48 hours after symptom onset. Every patient underwent an intensive diagnostic investigation. In 10/28 (36\%) of the TGA patients and $21 / 74(28 \%)$ of the TIA patients, DWI signal intensity changes occurred. The time to DWI and the duration of symptoms were comparable in TIA and TGA patients. Overall, TIA patients showed an increased prevalence of vascular risk factors compared with TGA patients. In the TGA group, patients with abnormal DWI showed carotid atherosclerosis significantly more frequently. Based on our data, we suggest that the aetiology of TGA could be explained by an ischaemic event; due to arterial thrombembolic ischaemia in one subgroup, particularly in those patients with increased vascular risk factors, and due to venous ischaemia in another subgroup with valsalva-like activities before symptom onset.
\end{abstract}

$\mathrm{T}$ he aetiology and pathogenesis of transient global amnesia (TGA) is still unclear. Several different causes such as transient ischaemic attack (TIA), seizure, migraine, and venous congestion with consecutive ischaemia of memory relevant structures have been discussed previously. ${ }^{1}$ Several investigators have used diffusion weighted imaging (DWI) to further examine the aetiology of TGA and reported controversial results. ${ }^{2-4}$ Delayed DWI abnormalities were reported in $100 \%$ of a TGA population. ${ }^{5}$ Interestingly, DWI abnormalities were also reported in $21-67 \%$ of TIA. ${ }^{6-8}$ As none of the recent studies evaluated patients with acute TIA and TGA using early DWI, we prospectively investigated the prevalence of DWI signal intensities and vascular risk factors in patients with acute TGA compared with those with TIA.

\section{METHODS}

Between June 2000 and June 2003, 256 TIA patients were treated at the stroke unit of the Department of Neurology. In 74 patients, a DWI was performed within 48 hours after symptom onset. All of these patients have been included (mean age 62.4 years; 95\% confidence interval (CI) 58.5 to 66.3). We also treated 35 patients with proven pure TGA between January 2000 and September 2003. In 28 of these patients ( 13 men, mean age 61.5 years; $95 \%$ CI 57.5 to 65.5 ), a DWI was performed within 48 hours of symptom onset.
TGA was diagnosed strictly according to well established criteria. ${ }^{10}$ TIA was defined as an acute transient focal neurological deficit caused by vascular disease that reversed totally within 24 hours. ${ }^{11}$ All TIA and TGA patients underwent an intensive diagnostic investigation including physical and neurological examination, duplex sonography of the carotid arteries, transcranial dopplersonography, 12 lead ECG, transthoracic echocardiography, and analysis of cardiovascular risk factors such as body mass index, prevalence of smoking, hypercholesterolaemia, arterial hypertension, diabetes mellitus, ischaemic heart disease, and carotid atherosclerosis. We also determined the intima-media thickness (IMT) of the common carotid artery, as described previously. ${ }^{12}$ The plaque score (PS) was calculated by summing the maximum thickness (in $\mathrm{mm}$ ) of each plaque located in bilateral carotid arteries as previously described by Nagai et al. ${ }^{13}$ In TGA patients, we performed an EEG to rule out an epileptic seizure.

\section{MRI measurements}

In TGA patients, transversal $\mathrm{T} 1$ and $\mathrm{T} 2$ sequences, and two DWI sequences (usually a transversal plane aligned to the course of the hippocampus and a coronal sequence angulated perpendicular to the hippocampus) were obtained with a 1.5 Telsa MRI (Magnetom Symphony; Siemens Medical Systems, Erlangen, Germany). The DWI imaging parameters have been described in detail previously. ${ }^{14}$ In TIA patients we performed conventional T1 and T2 weighted sequences, as well as at least one transversal DWI sequence using the same parameters, without angulation to the hippocampus. A DWI scan was considered positive if the hyperintensive region was confirmed in a second coronal or sagittal plane and if the scan revealed an area of hypointensity on the apparent diffusion coefficient (ADC) map.

The regions of hyperintensity on the $b=1000$ image and hypodensity on the ADC images were analysed by manually outlining the regions of interest using an image analysing system (Sigma Scan Pro; SPSS, Chicago, IL, USA) as described previously. ${ }^{14}$ Additionally we analysed the ratio between the lesion and corresponding normal contralateral tissue average signal intensity $(\mathrm{rAI})$ on $\mathrm{b}=1000\left(\mathrm{rAI}_{\mathrm{b}}=1000\right)$ and ADC images ( $\mathrm{rAI}$ ADC). The images were additionally analysed by a blinded neuroradiologist (MR).

\section{Statistical analysis}

All values are given as mean (95\% CI). Between group comparisons were performed with Fisher's exact test and Student's $t$ test for normally distributed data. For nonnormally distributed data, the Wilcoxon test was used.

Abbreviations: $A D C$, apparent diffusion coefficient; $D W I$, diffusion weighted imaging; IMT, intima-media thickness; PS, plaque score; TGA, transient global amnesia; TIA, transient ischaemic attack 
Values of $p<0.05$ were considered significant. For assessing the inter-rater agreement, we used kappa values.

\section{RESULTS}

On DWI, 21/74 TIA patients (28\%) and 10/28 TGA patients (36\%) showed signal intensity changes. The time to DWI was comparable in both groups. In TGA patients, all the lesions were located in memory relevant structures such as the hippocampus, the gyrus parahippocampalis, and the medial temporal lobe. Fig 1 gives an example of a typical DWI lesion in the TGA group. Of the TGA patients, 17/28 (54\%) reported valsalva-like activities, and 10 (36\%) described extraordinary emotional stress at the beginning of symptoms. TGA patients with hyperintensive DWI lesions (TGA+) showed an increased incidence of vascular risk factors such as hypertension, hypercholesterolaemia, and smoking, an increased IMT ( $1.03 \mathrm{~mm} v 0.76 \mathrm{~mm} ; \mathrm{p}=0.002)$ ) ) and a higher prevalence of carotid plaques $(70 \% \vee 17 \%, \mathrm{p}=0.01)$ compared with those without lesions (TGA-). In addition, the duration of symptoms $(4.3 \mathrm{~h} v 7.2 \mathrm{~h})$ and the time to DWI $(26.1 \mathrm{~h} v$ $19.7 \mathrm{~h}$ ) did not differ significantly between the TGA subgroups.

As expected, the vascular risk profile was different in TIA and TGA patients (table 1 ). The $\mathrm{rAI}_{\mathrm{b}=1000}$ was comparable in TIA and TGA patients, whereas the rAI ADC was significantly higher in TGA than in TIA patients $(0.91 \vee 0.85 ; \mathrm{p}=0.001$; table 1). Analysing the subgroup of TIA and TGA patients with abnormal DWI (TIA+ and TGA+), we detected com-parable risk factors in both groups. TIA patients showed a significant increased PS (7.53 $\mathrm{mm} v 4.13 \mathrm{~mm}, \mathrm{p}=0.02)$. The time to DWI was significantly shorter in TIA+ patients compared with TGA+ patients (14.6 h (95\% CI 8.0 to 21.1 ) $v$ $26.1 \mathrm{~h}$ (16.6 to 35.6); $\mathrm{p}=0.03)$. The kappa value for interrater agreement was 0.85 (0.64 to 1.05$)$, indicating very good reliability.

\section{DISCUSSION}

One of the hypotheses for the aetiology of TGA is transient cerebral ischaemia. In contrast to the findings in TIA patients, the results of DWI studies in TGA patients are controversial. There are several case reports describing DWI signal intensity changes in TGA patients, ${ }^{15-17}$ whereas two larger studies found no DWI abnormalities in TGA patients. ${ }^{3}$ In contrast, Sedlaczek et $a l^{5}$ previously described small parenchymal hyperintensities on DWI on day 2 in $11 / 11$ patients with clinically identified TGA in a serial DWI study. Hence, the investigators conclude that the aetiology of TGA is a primarily ischaemic event. In our study, we observed a similar prevalence of DWI lesions in TGA and TIA patients. The different prevalence of DWI lesions in our study compared with that of Sedlaczek et $a l^{5}$ might be explained by a later onset of DWI lesions in TGA patients. Interestingly, in the subgroup of TGA+ patients the time to DWI was $26 \mathrm{~h}$ compared with $19 \mathrm{~h}$ in the TGA- group, which may support this argument. As we did not perform a serial DWI study, we cannot rule out the possibility that additionally DWI lesions
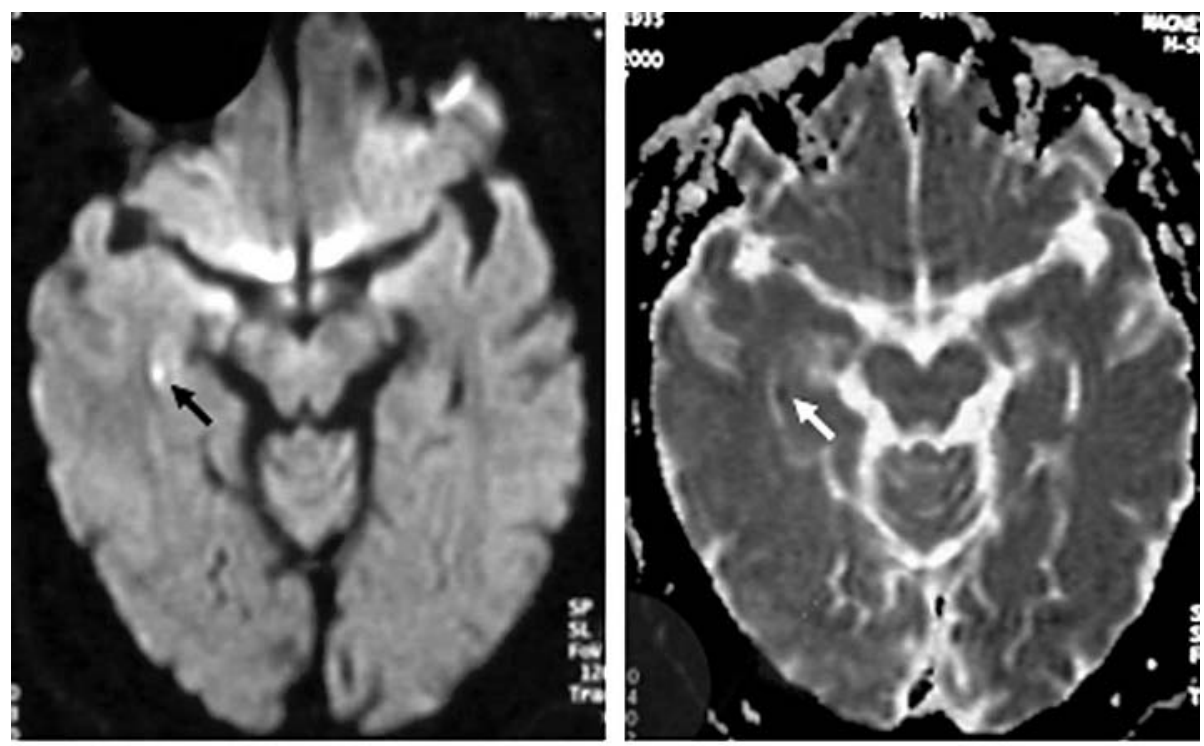

Figure 1 Transversal and coronal DWI and the calculated ADC images of a patient with transient global amnesia, performed 26 hours after symptom onset. The arrow indicates the signal intensity change.
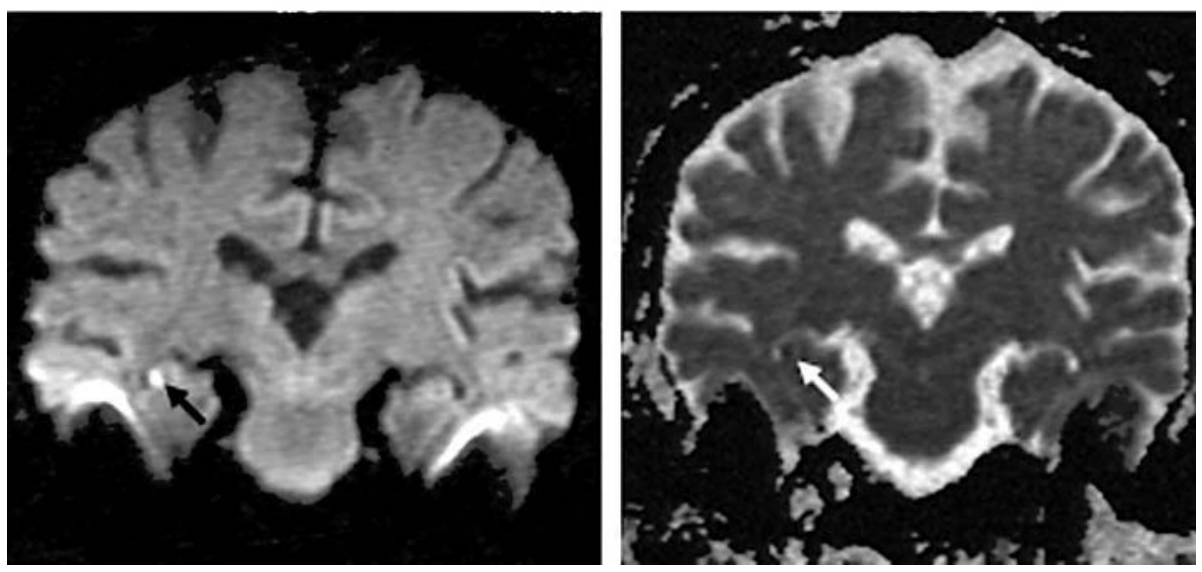
Table 1 Demographic data and cardiovascular risk factors of all TIA and TGA patients

\begin{tabular}{llll}
\hline & TGA & TIA & p \\
\hline No. of patients & 28 & 74 & $\mathrm{NS}$ \\
Age (years) & $61.5(57.5$ to 65.5$)$ & $62.4(58.5$ to 66.3$)$ & $\mathrm{NS}$ \\
Sex (male/female) & $13 / 15$ & $47 / 27$ & $\mathrm{NS}$ \\
Hypertension (n) & $17(61 \%)$ & $52(70 \%)$ & 0.06 \\
Ischaemic heart disease (n) & $1(4 \%)$ & $15(20 \%)$ & $\mathrm{NS}$ \\
Hypercholesterolaemia (n) & $12(43 \%)$ & $25(34 \%)$ & 0.01 \\
Smoking (n) & $5(18 \%)$ & $34(46 \%)$ & 0.01 \\
Diabetes (n) & $0(0 \%)$ & $15(20 \%)$ & $\mathrm{NS}$ \\
Atrial fibrillation (n) & $2(7 \%)$ & $6(8 \%)$ & 0.001 \\
IMT (mm) & $0.85(0.77$ to 0.92$)$ & $0.89(0.83$ to 0.98$)$ & 0.03 \\
Plaque score (mm) & $3.6(2.2$ to 4.9$)$ & $6.5(5.7$ to 7.3$)$ & $\mathrm{NS}$ \\
Carotid atherosclerosis (n) & $10(36 \%)$ & $46(62 \%)$ & $\mathrm{N}$ \\
Time to DWI (hours) & $21.7(17.4$ to 26.0$)$ & $16.7(13.6$ to 19.8$)$ & $\mathrm{NS}$ \\
Duration of symptoms (hours) & $6.1(4.3$ to 8.0$)$ & $6.2(3.9$ to 8.2$)$ & 0.001 \\
Lesion size (mm $)$ & $10.2(5.2$ to 15.1$)$ & $191(36$ to 266$)$ & \\
rAl b=1000 & $1.20(1.12$ to 1.28$)$ & $1.18(1.15$ to 1.20$)$ & \\
rAl ADC & $0.91(0.86$ to 0.97$)$ & $0.85(0.83$ to 0.88$)$ & \\
\hline Values in parentheses are 95\% confidence intervals unless otherwise indicated. & \\
\hline
\end{tabular}

could have been detected in later images. Owing to the comparable incidence of DWI lesions in TGA and TIA patients, we further evaluated the vascular risk profile of TGA and TIA patients, particularly of the subgroup with and without pathological DWI, in more detail. As expected, we found a lower incidence of vascular risk factors in TGA patients compared with TIA patients. Interestingly, TGA+ patients showed a higher vascular risk profile, significantly more frequent carotid atherosclerosis, and an increased IMT than did TGA- patients. These findings may support, particularly in this subgroup of TGA patients, an arterial embolic event. Nevertheless, there are several points that question an arterial ischaemic aetiology in all TGA patients: the different cardiovascular risk profile, the better long term outcome, and the low recurrence rate. ${ }^{9} 1819$

Lewis ${ }^{1}$ pointed out that TGA might be caused by venous congestion as a consequence of a retrograde transmission of high venous pressure after valsalva-like activities. This hypothesis was confirmed by the finding of a higher incidence of a retrograde flow pattern in the internal jugular vein in TGA. ${ }^{20-22}$ Others have proposed a specific anxious personality trait in TGA patients, which might induce vasoconstriction due to hyperventilation during an emotional arousal. ${ }^{23}$ Therefore, temporary hypoperfusion either due to venous congestion or vasoconstriction due to hyperventilation might cause TGA. The increased vulnerability of the hippocampus and the venous drainage might help to explain why memory structures are first involved..$^{22}$ As DWI lesions in venous infarction are more heterogeneous, ${ }^{24}$ it is conceivable that DWI lesions are underestimated in patients with venous ischaemia. Interestingly, the ADC ratio was significantly higher in TGA patients than in TIA patients. Increased diffusion does not necessary mean ischaemia, and it occurs even in multiple sclerosis and seizure. However, ADC changes seen during post-ictal DWI are complex, including generalised ADC changes, cortical hyperdensities, no major changes, and even ADC increase. ${ }^{25}$ The lesions we found in our population were all quite similar and comparable with DWI changes in TIA patients. Additionally, if the DWI lesions in our study were due to epileptic changes, it might be postulated that at least in a few TGA patients, EEG changes should occur.

Based on our data it might be speculated that transient ischaemia, either embolic or due to venous congestion in memory relevant structures, cause TGA and that the transient abnormalities in brain function might be too small to be detected in every TGA patient using DWI. In both groups, emotional distress might act as a pathogenic cofactor.

\section{Authors' affiliations}

K Winbeck, T Etgen, D Sander, Department of Neurology, Technical University of Munich, Germany

H G von Einsiedel, M Rö̈tinger, Department of Neuroradiology, Technical University of Munich, Germany

Competing interests: none declared

Correspondence to: Dr K Winbeck, Department of Neurology, Technical University of Munich, Möhlstr. 28, 81675 München; kerstin.winbeck@ neuro.med.tu-muenchen.de

Received 31 March 2004

In revised form 21 May 2004

Accepted 24 June 2004

\section{REFERENCES}

1 Lewis SL. Aetiology of transient global amnesia. Lancet 1998;352:397-9.

2 Strupp M, Bruning R, Wu RH, et al. Diffusion-weighted MRI in transient global amnesia: elevated signal intensity in the left mesial temporal lobe in 7 of 10 patients. Ann Neurol 1998:43:164-70.

3 Gass A, Gaa J, Hirsch J, et al. Lack of evidence of acute ischemic tissue change in transient global amnesia on single-shot echo-planar diffusion-weighted MRI. Stroke 1999;30:2070-2.

4 Huber R, Aschoff AJ, Ludolph AC, et al. Transient global amnesia. Evidence against vascular ischemic etiology from diffusion weighted imaging. I Neurol 2002;249:1520-4.

5 Sedlaczek O, Hirsch J, Grips E, et al. Delayed hippocampal DWI changes are associated with transient global amnesia. Cerebrovascular Dis 2002;13(Suppl 3):58.

6 Kidwell CS, Alger JR, Di Salle F, et al. Diffusion MRI in patients with transient ischemic attacks. Stroke 1999;30:1174-80.

7 Rovira A, Rovira-Gols A, Pedraza S, et al. Diffusion-weighted MR imaging in the acute phase of transient ischemic attacks. AJNR Am J Neuroradiol 2002;23:77-83.

8 Crisostomo RA, Garcia MM, Tong DC. Detection of diffusion-weighted MRI abnormalities in patients with transient ischemic attack: correlation with clinical characteristics. Stroke 2003;34:932-7.

9 Hodges JR, Warlow CP. The aetiology of transient global amnesia. A casecontrol study of 114 cases with prospective follow-up. Brain 1990;113:639-57.

10 Caplan LR. Transient global amnesia. Amsterdam: Elsevier Science Publishers, 1985.

11 Special report from the National Institute of Neurological Disorders and Stroke. Classification of cerebrovascular diseases III. Stroke 1990;21:637-76.

12 Sander D, Winbeck K, Klingelhofer J, et al. Enhanced progression of early carotid atherosclerosis is related to Chlamydia pneumoniae (Taiwan acute respiratory) seropositivity. Circulation 2001;103:1390-5.

13 Nagai Y, Kitagawa K, Yamagami H, et al. Carotid artery intima-media thickness and plaque score for the risk assessment of stroke subtypes. Ultrasound Med Biol 2002;28:1239-43.

14 Winbeck K, Bruckmaier K, Etgen T, et al. Transient ischemic attack and stroke can be differentiated by analyzing early diffusion-weighted imaging signal intensity changes. Stroke 2004;35:1095-9.

15 Inamura T, Nakazaki K, Yasuda O, et al. (A lesion diagnosed by MRI in a case of transient global amnesia). No To Shinkei 2002;54:419-22.

16 Matsui M, Imamura T, Sakamoto S, et al. Transient global amnesia: increased signal intensity in the right hippocampus on diffusion-weighted magnetic resonance imaging. Neuroradiology 2002;44:235-8. 
17 Greer DM, Schaefer PW, Schwamm LH. Unilateral temporal lobe stroke causing ischemic transient global amnesia: role for diffusion-weighted imaging in the initial evaluation. J Neuroimaging 2001;11:317-19.

18 Gandolfo C, Caponnetto C, Conti M, et al. Prognosis of transient global amnesia: a long-term follow-up study. Eur Neurol 1992;32:52-7.

19 Zorzon M, Antonutti L, Mase G, et al. Transient global amnesia and transient ischemic attack. Natural history, vascular risk factors, and associated conditions. Stroke 1995;26:1536-42.

20 Sander D, Winbeck K, Etgen T, et al. Disturbance of venous flow patterns in patients with transient global amnesia. Lancet 2000;356:1982-4.
21 Akkawi NM, Agosti C, Rozzini L, et al. Transient global amnesia and disturbance of venous flow patterns. Lancet 2001;357:957.

22 Maalikjy Akkawi N, Agosti C, et al. Transient global amnesia: a clinical and sonographic study. Eur Neurol 2003;49:67-71.

23 Pantoni L, Lamassa $M$, Inzitari D. Transient global amnesia: a review emphasizing pathogenic aspects. Acta Neurol Scand 2000;102:275-83.

24 Ducreux D, Petit-Lacour MC, Benoudiba F, et al. Diffusion-weighted imaging in a case of Wernicke encephalopathy. J Neuroradiol 2002;29:39-42.

25 Hufnagel A, Weber J, Marks S, et al. Brain diffusion after single seizures. Epilepsia 2003;44:54-63.

HISTORICAL NOTE

\section{Gabriel Anton's (1858-1933) contribution to the history of neurosurgery}

doi: 10.1136/jnnp.2004.048058

$\mathrm{T}$ he existence of neurosurgery as a scientific discipline, owes its emergence in large to surgeons themselves as

well as to neurologists and psychiatrists who utilised the morphological approach. Among the lead contributors to nascent neurosurgery was the neuro-psychiatrist Gabriel Anton, who strongly influenced the development of this discipline during the first two decades of the 20th century. One of his most renowned scientific achievements was the Anton-von Bramannsche Balkenstich method of treating hydrocephalus. In collaboration with fellow surgeons Gustav von Bramann and Victor Schmieden, he proposed new clinical procedures for the treatment of hydrocephalus: the Balkenstich method and the suboccipital puncture.

\section{Preliminary considerations}

The establishment of neurosurgery as an independent discipline within the broad field of medicine required a fundamental knowledge of the structure and function of the nervous system. The beginning of modern neurosurgery is strongly associated with names such as Victor Horsley (18571916) in England, and Harvey Cushing (1869-1939) and Percival Bailey (1892-1973) in the USA.

The pioneering German surgeon Ernst von Bergmann (1836-1907) insisted on the necessity of establishing a scientific base for neurosurgery. Above all other aspects of neuroanatomical knowledge, clinical practice required expertise in neurological diagnostic methodology. This necessity gave birth to an era of interdependent cooperation between surgeons and neurologists. An excellent example of this fruitful alliance can be found in the beginning of the 20th century at the University of Halle, where many advancements in the field of neurosurgery were coupled with the names of surgeons such as Fedor Krause (1857-1937) and Gustav von Bramann (1854-1913), as well as Gabriel Anton.

\section{Gabriel Anton - life and scientific works'}

Gabriel Anton was born on August 28, 1858 in Saaz (Bohemia). On completion of his medical education in Prague, he worked as an assistant to Arnold Pick. In 1887 he resigned his position in order to join Theodor Meynert (1833-1892) in Vienna. Meynert was a very important representative of neuroanatomy and neuropathology at this time.

In 1905 Anton received a full professorship in psychiatry at the University of Halle in Germany. He held the chair of psychiatry at the department of psychiatry until his Emeritus in 1926. Gabriel Anton passed away on January 3, 1933.

Anton's initial inspiration for his scientific activities derived from the ideas of Arnold Pick (1851-1924) and Hans Chiari (1851-1916). His early scientific work dealt with anatomical examinations of brain structure and developmental disorders of the brain. In 1895 he described the neurological and psychological symptoms that result from high cerebral pressure. In certain cases he insisted on the importance of early operative procedures with trepanation for the release of cerebral pressure. ${ }^{2}$ His main focus of interest laid on problems arising from hydrocephalus and cerebral oedema. These workings formed the basis for his later theories that in turn impacted the field of neurosurgery.

\section{Anton's influence on brain surgery}

Anton, in collaboration with the surgeon Gustav von Bramann, developed the Balkenstich method (callosal puncture) - an operational procedure that applies a form of ventricle drainage to reduce cerebral pressure. ${ }^{3}$ Anton proposed a puncture of the ventricle made through a direct path from the corpus callosum (ventriculopuncture) to allow drainage into the subarachnoid space without implant. Unlike previous methods that required repeated draining procedures, Anton's drainage procedure took place only once. Thus, the Balkenstich method provided an understanding of cerebrospinal fluid (CSF) drainage and avoided the negative side effects accompanying the necessity to continually drain fluids from the brain. The Balkenstich method was primarily recommended to be applied to release pressure in case of hydrocephalus. In the following years the Balkenstich method was introduced in clinics around the world.

In 1917, a new cooperation with Bramann's heir, Victor Schmieden (1874-1945), produced a second major surgical technique for releasing pressure on the brain, known as the suboccipital puncture. ${ }^{4}$ This procedure was the puncture of the membrane atlantooccipitalis resulting in a continuous drainage of CSF to the neck musculature.

In 1938 Arne Torkildsen (1899-1968) published his works on ventriculocisternostomy. This "Torkildsen shunt" proved to be very successful in the treatment of hydrocephalus and produced repeated, more permanent successes. The Balkenstich method was finally superseded by Torkildsen's technique.

E Kumbier, K Haack

Department of Psychiatry and Psychotherapy, University Rostock, Germany

Correspondence to: Ekkehardt Kumbier, Department of Psychiatry and Psychotherapy, University Rostock, Germany, Gehlsheimer Straße 20, D-18147 Rostock; ekkehardt.kumbier@medizin.uni-rostock.de

\section{References}

1 Kumbier E, Haack K. Gabriel Anton (1858-1933). Comments on the issue cover picture. Nervenarzt 2002;73:201-2.

2 Anton G. Über die Folgen der Raumbeengung im Schädel für das Nervensystem. Mittheilungen des Vereines der Ärzte in Steiermark 1895:32:85-90.

3 Anton G, von Bramann FG. Balkenstich bei Hydrozephalien, Tumoren und bei Epilepsie. Münch med Wschr 1908;55:1673-7.

4 Anton G, Schmieden V. Der Subokzipitalstich; eine neve druckentlastende Hirnoperationsmethode. Arch Psychiat Nervenkr 1917;58:1-30. 